\title{
Analytical Solutions for Systems of Singular Partial Differential-Algebraic Equations
}

\author{
U. Filobello-Nino, ${ }^{1}$ H. Vazquez-Leal, ${ }^{1}$ B. Benhammouda, ${ }^{2}$ A. Perez-Sesma, ${ }^{1}$ \\ V. M. Jimenez-Fernandez, ${ }^{1}$ J. Cervantes-Perez, ${ }^{1}$ A. Sarmiento-Reyes, ${ }^{3}$ \\ J. Huerta-Chua, ${ }^{4}$ L. J. Morales-Mendoza, ${ }^{5}$ M. Gonzalez-Lee, ${ }^{5}$ A. Diaz-Sanchez, ${ }^{3}$ \\ D. Pereyra-Díaz, ${ }^{1}$ and R. López-Martínez ${ }^{6}$ \\ ${ }^{1}$ Facultad de Instrumentación Electrónica, Universidad Veracruzana, Circuito Gonzalo Aguirre Beltrán S/N, \\ 91000 Xalapa, VER, Mexico \\ ${ }^{2}$ Higher Colleges of Technology, Abu Dhabi Men's College, P.O. Box 25035, Abu Dhabi, UAE \\ ${ }^{3}$ Instituto Nacional de Astrofísica, Óptica y Electrónica, Luis Enrique Erro 1, 72840 Santa María Tonantzintla, PUE, Mexico \\ ${ }^{4}$ Facultad de Ingeniería Civil, Universidad Veracruzana, Venustiano Carranza S/N, Colonia Revolución, \\ 93390 Poza Rica, VER, Mexico \\ ${ }^{5}$ Departamento de Ingeniería Electrónica, Universidad Veracruzana, Venustiano Carranza S/N, \\ Colonia Revolución, 93390 Poza Rica, VER, Mexico \\ ${ }^{6}$ Facultad de Matemáticas, Universidad Veracruzana, Circuito Gonzalo Aguirre Beltrán S/N, 91000 Xalapa, VER, Mexico
}

Correspondence should be addressed to H. Vazquez-Leal; hvazquez@uv.mx

Received 13 October 2014; Accepted 17 December 2014

Academic Editor: Baodong Zheng

Copyright (C) 2015 U. Filobello-Nino et al. This is an open access article distributed under the Creative Commons Attribution License, which permits unrestricted use, distribution, and reproduction in any medium, provided the original work is properly cited.

This paper proposes power series method (PSM) in order to find solutions for singular partial differential-algebraic equations (SPDAEs). We will solve three examples to show that PSM method can be used to search for analytical solutions of SPDAEs. What is more, we will see that, in some cases, Padé posttreatment, besides enlarging the domain of convergence, may be employed in order to get the exact solution from the truncated series solutions of PSM.

\section{Introduction}

The importance of research on partial differential-algebraic equations (PDAEs) is that they are used in the mathematical modeling of many phenomena, both practical and theoretical. These systems arise, for example, in nanoelectronics, electrical networks, and mechanical systems, among many others. Despite the importance of this topic, it may be considered relatively new and little known.

Although the case of constant-coefficient linear PDAEs has been investigated by means of numerical methods, for instance, in $[1,2]$, perhaps the more relevant aspect of PDAEs, both linear and nonlinear, is the concept of index. The differentiation index is defined as the minimum number of times that all or part of the PDAEs must be differentiated with respect to time, in order to obtain the time derivative of the solution, as a continuous function of the solution and its space derivatives [3]. A fact that justifies the search for other methods of solution to these equations is that the solutions of higher index PDAEs (index greater than one) become very complicated, even for numerical methods, and many application problems lead to PDAEs with different indices. A further difficulty to be considered that arises and affects also other kinds of systems of differential equations, as well as differential equations, is the presence of singularities, which are related to points at which some terms of the differential equations become infinite or undefined.

In recent years, several methods focused on approximating nonlinear and linear problems, as an alternative to classical methods, have been reported, such as those based on variational approaches [4-7], tanh method [8], exp-function $[9,10]$, Adomian's decomposition method [11-16], parameter 
expansion [17], homotopy perturbation method [7, 16, 1846], homotopy analysis method [47], homotopy asymptotic method [48], series method [49, 50], and perturbation method [51-54], among many others. Also, a few exact solutions to nonlinear differential equations have been reported occasionally [55].

This study shows that power series method (PSM) [56,57] is able to address the above difficulties to obtain power series solutions for singular partial differential-algebraic equations (SPDAEs), that is, PDAEs with singular points. These systems turn out to be difficult even for numerical methods. More generally, we will see that the combination of PSM and Padé posttreatment could be effective to improve the PSM's truncated series solutions in convergence rate; what is more, sometimes it ends up giving the exact solution of the system, such as what will happen in our third case study.

This paper is organized as follows. In Section 2, we introduce the basic idea of power series method. Section 3 provides a brief explanation of application of PSM to solve SPDAEs. Section 4 presents three case studies: one singular nonlinear index-one system, one singular linear indextwo system, and one singular nonlinear index-two system. Besides, a discussion on the results is presented in Section 5. Finally, a brief conclusion is given in Section 6.

\section{Basic Concept of Power Series Method}

It can be considered that a nonlinear differential equation can be expressed as

$$
A(u)-f(t)=0, \quad t \in \Omega,
$$

with the following boundary condition:

$$
B\left(u, \frac{\partial u}{\partial n}\right)=0, \quad t \in \Gamma,
$$

where $A$ is a general differential operator, $B$ is a boundary operator, $f(t)$ is a known analytical function, and $\Gamma$ is the domain boundary for $\Omega$.

PSM $[49,50]$ assumes that the solution of a differential equation can be written in the following form:

$$
u(t)=\sum_{n=0}^{\infty} u_{n} t^{n}
$$

where $u_{0}, u_{1}, \ldots$ are unknown functions to be determined by series method.

The method of solution for differential equations can be summarized as follows.

(1) Equation (3) is substituted into (1), and then we regroup the resulting polynomial equation in terms of powers of $t$.

(2) We equate each coefficient of the above-mentioned polynomial to zero.

(3) As a consequence, a linear algebraic system for the unknowns of (3) is obtained.

(4) To conclude, the solution of the above system allows obtaining the coefficients $u_{0}, u_{1}, \ldots$.

\section{Application of PSM to Solve PDAE Systems}

Since many applications problems in science and engineering are often modeled by semiexplicit PDAEs, we consider therefore the following class of PDAEs:

$$
\begin{gathered}
u_{1 t}=\phi\left(u, u_{x}, u_{x x}\right), \\
0=\psi\left(u, u_{x}, u_{x x}\right), \quad(t, x) \in(0, T) \times(a, b),
\end{gathered}
$$

where $u_{k}:[0, T] \times[a, b] \rightarrow R^{m_{k}}, k=1,2$, and $b>a$; in other words $u=\left(u_{1}, u_{2}\right)$.

For clarification, the method is described for the general system (4)-(5) where the number of unknowns is given by $m_{1}+m_{2}$. In this notation, $u_{1}$ (differential unknown) has $m_{1}$ components and $u_{2}$ (algebraic unknown) has $m_{2}$ components. In fact $m_{1}$ and $m_{2}$ can take any values greater than or equal to one, so that the number of unknowns in (4)(5) is greater than or equal to 2 .

System (4)-(5) is subject to the initial condition

$$
u_{1}(0, x)=g(x), \quad a \leq x \leq b,
$$

and some suitable boundary conditions

$$
B\left(u(t, a), u(t, b), u_{x}(t, a), u_{x}(t, b)\right)=0, \quad 0 \leq t \leq T,
$$

where $g(x)$ is a given function.

We assume that the solution to initial boundary-value problem (4)-(7) exists and is unique and sufficiently smooth.

To simplify the exposition of the PSM, we integrate first (4) with respect to $t$ and use the initial condition (6) to obtain

$$
u_{1}(t, x)-g(x)-\int_{0}^{t} \phi\left(u, u_{x}, u_{x x}\right) d t=0 .
$$

It is important to note that the time integration of (4) is not relevant to the solution procedure presented here, so one can apply the PSM directly to (4).

A fact that justifies the use of PSM is that, in general terms, getting solutions for PDAEs becomes very complicated, even for numerical methods. Moreover, there are not systematic analytical or numerical methods to solve these problems.

In view of PSM, we assume the solution components $u_{k}(t, x), k=1,2$, have the form

$$
u_{k}(t, x)=u_{k, 0}(x)+u_{k, 1}(x) t+u_{k, 2}(x) t^{2}+\cdots,
$$

where $u_{k, n}(x), k=1,2, n=0,1,2, \ldots$, are unknown functions to be determined later on by the PSM.

Then substitute (9) into system (4)-(5) and equate the coefficients of powers of $t$ in the resulting equation to zero, to obtain an algebraic linear system for the coefficients, whose solution is employed in (9), with the end of obtaining a solution for (4)-(7) in series form. These series may have limited regions of convergence, even if we take a large number of terms. Therefore, in some cases, it will be convenient to apply the Padé resummation method to PSM truncated series to enlarge the convergence region as depicted in the next section. A relevant fact is that the steps outlined in this section will be also sufficient to obtain satisfactory solutions for the most difficult case of SPDAEs. 


\section{Case Studies}

The objective of this section is employing PSM, in order to solve three SPDAE systems.

Our results will show the efficiency of the presented method.

4.1. Nonlinear Index-One SPDAE (following Section 3, $m_{1}=1$ and $\left.m_{2}=1\right)$. Consider the following:

$$
\begin{gathered}
u_{1 t}-u_{1 x x}+u_{1} u_{1 x}+\frac{u_{2}}{x}=x^{3}-6 x t+3 t^{2} x^{5}+\frac{t^{4}}{x^{2}} \\
u_{1}+u_{2}=t x^{3}+\frac{t^{4}}{x}, \quad t>0,
\end{gathered}
$$

subject to the initial conditions

$$
u_{1}(0, x)=0 .
$$

In order to apply PSM, we integrate (10) with respect to $t$ and use the initial condition (12) to obtain

$$
\begin{aligned}
& u_{1}(t, x) \\
& \quad=\int_{0}^{t}\left[u_{1 x x}-u_{1} u_{1 x}-\frac{u_{2}}{x}+x^{3}-6 x t+3 t^{2} x^{5}+\frac{t^{4}}{x^{2}}\right] d t .
\end{aligned}
$$

PSM assumes that $u(t, x)$ and $v(t, x)$ can be written as

$$
\begin{aligned}
& u_{1}(t, x)=u_{10}(x)+u_{11}(x) t+u_{12}(x) t^{2}+\cdots, \\
& u_{2}(t, x)=u_{20}(x)+u_{21}(x) t+u_{22}(x) t^{2}+\cdots,
\end{aligned}
$$

where $u_{10}(x), u_{11}(x), u_{12}(x), u_{20}(x), u_{21}(x), u_{22}(x), \ldots$ are unknown functions.

This case study is simplified, substituting (14) and (15) into (11), to get

$$
\sum_{n=0}^{\infty} u_{2 n} t^{n}=\frac{t^{4}}{x}+t x^{3}-\sum_{n=0}^{\infty} u_{1 n} t^{n}
$$

On the other hand, substituting (14) through (16) into (13) leads to

$$
\begin{aligned}
\sum_{n=0}^{\infty} u_{1 n} t^{n}=\int_{0}^{t}\left[\sum_{n=0}^{\infty} u_{1 n}^{\prime \prime} t^{n}-\sum_{n=0}^{\infty} \sum_{m=0}^{\infty} u_{1 n} u_{1 m}^{\prime} t^{n+m}\right. \\
-\frac{1}{x}\left(\frac{t^{4}}{x}+t x^{3}-\sum_{n=0}^{\infty} u_{1 n} t^{n}\right) \\
\left.+x^{3}-6 x t+3 t^{2} x^{5}+\frac{t^{4}}{x^{2}}\right] d t
\end{aligned}
$$

From here on, the dash notation in $u^{\prime}$ denotes the ordinary derivative with respect to $x$.

Integrating the above result, it is obtained that

$$
\begin{aligned}
\sum_{n=0}^{\infty} u_{1 n} t^{n}= & \sum_{n=0}^{\infty} u_{1 n}^{\prime \prime} \frac{t^{n+1}}{n+1}-\sum_{n=0}^{\infty} \sum_{m=0}^{\infty} u_{1 n} u_{1 m}^{\prime} \frac{t^{n+m+1}}{n+m+1} \\
& -\frac{x^{2} t^{2}}{2}+x^{3} t-3 x t^{2}+x^{5} t^{3}+\frac{1}{x} \sum_{n=0}^{\infty} \frac{u_{1 n} t^{n+1}}{n+1} .
\end{aligned}
$$

Standardizing the summation index and grouping, we get the recursive formula

$$
\begin{aligned}
& u_{10} t^{0}-x^{3} t+\left(3 x+\frac{x^{2}}{2}\right) t^{2}-x^{5} t^{3} \\
& +\sum_{k=1}^{\infty}\left[u_{1 k}-\frac{u_{1 k-1}^{\prime \prime}}{k}+\sum_{m=0}^{\infty} \frac{u_{1 k-m-1} u_{1 m}^{\prime}}{k}-\frac{u_{1 k-1}}{k x}\right] t^{k}=0
\end{aligned}
$$

Equating the coefficients of powers of $t$ to zero in (19), we obtain

$$
\begin{gathered}
k=0, \\
u_{0}=0, \\
k=1, \\
u_{11}=u_{10}^{\prime \prime}-u_{10}^{\prime} u_{10}+\frac{1}{x} u_{10}+x^{3} ;
\end{gathered}
$$

after employing (20), it is obtained that

$$
\begin{gathered}
u_{11}=x^{3}, \\
k=2,
\end{gathered}
$$

$$
u_{12}=\frac{u_{11}^{\prime \prime}}{2}-\frac{u_{10}^{\prime} u_{11}}{2}-\frac{u_{11}^{\prime} u_{10}}{2}+\frac{u_{11}}{2 x}-\frac{x^{2}}{2}-3 x
$$

substituting (20) and (21) in the above equation, it is obtained that

$$
\begin{gathered}
u_{12}=0, \\
k=3, \\
u_{13}=\frac{u_{12}^{\prime \prime}}{3}-\frac{u_{10}^{\prime} u_{12}}{3}-\frac{u_{11}^{\prime} u_{11}}{3}-\frac{u_{12}^{\prime} u_{10}}{3}+\frac{u_{12}}{3 x}+x^{5}
\end{gathered}
$$

after substituting (20), (21), and (22) in the last equation, we get

$$
\begin{gathered}
u_{13}=0, \\
k=4, \\
u_{14}=\frac{u_{13}^{\prime \prime}}{4}-\frac{u_{10}^{\prime} u_{13}}{4}-\frac{u_{11}^{\prime} u_{12}}{4}-\frac{u_{12}^{\prime} u_{11}}{4}-\frac{u_{13}^{\prime} u_{10}}{4}+\frac{u_{13}}{4 x} ;
\end{gathered}
$$

after employing (20), (21), (22), and (23), we get

$$
\begin{gathered}
u_{14}=0, \\
k=5, \\
u_{15}=\frac{u_{14}^{\prime \prime}}{5}-\frac{u_{10}^{\prime} u_{14}}{5}-\frac{u_{11}^{\prime} u_{13}}{5}-\frac{u_{12}^{\prime} u_{12}}{5} \\
-\frac{u_{13}^{\prime} u_{11}}{5}-\frac{u_{14}^{\prime} u_{10}}{5}+\frac{u_{14}}{5 x} ;
\end{gathered}
$$


the substitution of (20), (21), (22), (23), and (24) leads to

$$
u_{15}=0
$$

in the same way we obtain

$$
u_{16}=u_{17}=u_{18}=\cdots=0 .
$$

Substituting (20) through (26) into (14) leads us to

$$
u_{1}(t, x)=x^{3} t
$$

Finally, substituting (27) into (11) leads to

$$
u_{2}(t, x)=\frac{t^{4}}{x} .
$$

Thus, (27) and (28) are the exact solution for SPDAE system (10)-(12).

4.2. Linear Index-Two SPDAE with Variable Coefficients $\left(m_{1}=\right.$ 2, $m_{2}=1$ ). Consider the following:

$$
\begin{gathered}
u_{1 t}=x^{2} u_{1 x x}-3 u_{1}+u_{3}+\frac{x^{2}}{1+t}, \\
u_{2 t}=x^{2} u_{2 x x}-3 u_{2}+u_{3}+\frac{x^{2}}{1+t}, \\
0=u_{1}+u_{2}-2 x^{2} \ln (1+t),
\end{gathered}
$$

subject to the initial conditions

$$
\begin{aligned}
& u_{1}(0, x)=0, \quad u_{2}(0, x)=0, \\
& -1<t \leq 1, \quad-\infty<x<\infty .
\end{aligned}
$$

The integration of (29) and (30) with respect to $t$ and using the initial conditions (32) lead to

$$
\begin{aligned}
& u_{1}(t, x)=\int_{0}^{t}\left[x^{2} u_{1 x x}-3 u_{1}+u_{3}\right] d t+x^{2} \ln (1+t), \\
& u_{2}(t, x)=\int_{0}^{t}\left[x^{2} u_{2 x x}-3 u_{2}+u_{3}\right] d t+x^{2} \ln (1+t)
\end{aligned}
$$

assuming that $u_{1}(t, x), u_{2}(t, x)$, and $u_{3}(t, x)$ can be written as

$$
\begin{aligned}
& u_{1}(t, x)=u_{10}(x)+u_{11}(x) t+u_{12}(x) t^{2}+\cdots, \\
& u_{2}(t, x)=u_{20}(x)+u_{21}(x) t+u_{22}(x) t^{2}+\cdots, \\
& u_{3}(t, x)=u_{30}(x)+u_{31}(x) t+u_{32}(x) t^{2}+\cdots,
\end{aligned}
$$

where $\quad u_{10}(x), u_{11}(x), \ldots, u_{20}(x), u_{21}(x), \ldots, u_{30}(x), u_{31}(x)$, $\ldots$ are unknown functions to be determined later on by the PSM method.

After substituting (35) and (37) into (33), we get

$$
\begin{aligned}
u_{10} t^{0}+\sum_{k=1}^{\infty} \frac{1}{k}\left[k u_{1 k}-x^{2} u_{1 k-1}^{\prime \prime}+3 u_{1 k-1}\right. & \\
\left.-u_{3 k-1}-x^{2}(-1)^{k-1}\right] t^{k} & =0,
\end{aligned}
$$

where we have standardized the summation index and employed the following Taylor series expansion:

$$
\ln (1+t)=\sum_{n=1}^{\infty} \frac{(-1)^{n-1}}{n} t^{n}, \quad-1<t \leq 1
$$

In the same way, the substitution of (36) and (37) into (34) leads to

$$
\begin{aligned}
u_{20} t^{0}+\sum_{k=1}^{\infty} \frac{1}{k}\left[k u_{2 k}-x^{2} u_{2 k-1}^{\prime \prime}+3 u_{2 k-1}\right. & \\
- & \left.u_{3 k-1}-x^{2}(-1)^{k-1}\right] t^{k}=0 .
\end{aligned}
$$

On the other hand, after substituting (35), (36), and (39) into (31), we have

$$
\sum_{k=1}^{\infty}\left[u_{1 k}+u_{2 k}-\frac{2 x^{2}}{k}(-1)^{k-1}\right] t^{k}=0,
$$

where we have employed the following results, deduced from (38) and (40):

$$
u_{10}=u_{20}=0 \text {. }
$$

Equations (38), (40), and (41) give rise to the following formulas:

$$
\begin{gathered}
u_{1 n}=\frac{x^{2} u_{1 n-1}^{\prime \prime}-3 u_{1 n-1}+u_{3 n-1}+(-1)^{n-1} x^{2}}{n}, \quad n \geq 1, \\
u_{2 n}=\frac{x^{2} u_{2 n-1}^{\prime \prime}-3 u_{2 n-1}+u_{3 n-1}+(-1)^{n-1} x^{2}}{n}, \quad n \geq 1, \\
u_{1 n}+u_{2 n}=\frac{2 x^{2}(-1)^{n-1}}{n}, \quad n \geq 1 .
\end{gathered}
$$

Combining the result of adding (43) and (44), with (45), we obtain

$$
u_{3 n-1}=-\frac{1}{2}\left(u_{1 n-1}^{\prime \prime}+u_{2 n-1}^{\prime \prime}\right) x^{2}+\frac{3}{2}\left(u_{1 n-1}+u_{2 n-1}\right), \quad n \geq 1
$$

The substitution of (46) into (43) and (44), respectively, leads us to

$$
\begin{gathered}
u_{1 n}=\frac{1}{2 n}\left(x^{2} u_{1 n-1}^{\prime \prime}-3 u_{1 n-1}+3 u_{2 n-1}\right. \\
\left.-x^{2} u_{2 n-1}^{\prime \prime}+2(-1)^{n-1} x^{2}\right), \quad n \geq 1, \\
u_{2 n}=\frac{1}{2 n}\left(x^{2} u_{2 n-1}^{\prime \prime}-3 u_{2 n-1}+3 u_{1 n-1}\right. \\
\left.-x^{2} u_{1 n-1}^{\prime \prime}+2(-1)^{n-1} x^{2}\right), \quad n \geq 1 .
\end{gathered}
$$


From recursion formulas (46) and (47), we get the functions

$$
\begin{gathered}
u_{10}(x)=0, \quad u_{11}(x)=x^{2}, \quad u_{12}(x)=\frac{-x^{2}}{2}, \\
u_{13}=\frac{x^{2}}{3}, \quad u_{14}=\frac{-x^{2}}{4} \cdots, \\
u_{20}(x)=0, \quad u_{21}(x)=x^{2}, \quad u_{22}(x)=\frac{-x^{2}}{2}, \\
u_{23}=\frac{x^{2}}{3}, \quad u_{24}=\frac{-x^{2}}{4} \cdots, \\
u_{30}(x)=0, \quad u_{31}(x)=x^{2}, \quad u_{32}(x)=\frac{-x^{2}}{2}, \\
u_{33}=\frac{x^{2}}{3}, \quad u_{34}=\frac{-x^{2}}{4} \cdots .
\end{gathered}
$$

After substituting (48) through (50) into series (35), (36), and (37), respectively, we get

$$
\begin{aligned}
& u_{1}(t, x)=x^{2}\left[t-\frac{t^{2}}{2}+\frac{t^{3}}{3}-\frac{t^{4}}{4}+\cdots\right], \\
& u_{2}(t, x)=x^{2}\left[t-\frac{t^{2}}{2}+\frac{t^{3}}{3}-\frac{t^{4}}{4}+\cdots\right], \\
& u_{3}(t, x)=x^{2}\left[t-\frac{t^{2}}{2}+\frac{t^{3}}{3}-\frac{t^{4}}{4}+\cdots\right] .
\end{aligned}
$$

After identifying the $n$th terms of the series (51), (52), and (53) as $\left((-1)^{n-1} / n\right) t^{n}$, we conclude that

$$
\begin{aligned}
& u_{1}(t, x)=x^{2} \ln (1+t), \\
& u_{2}(t, x)=x^{2} \ln (1+t), \\
& u_{3}(t, x)=x^{2} \ln (1+t)
\end{aligned}
$$

which is the exact solution of (29)-(32) (see (39)).

4.3. Nonlinear Index-Two SPDAE with Variable Coefficients $\left(m_{1}=2, m_{2}=1\right)$. Finally, consider the following:

$$
\begin{gathered}
u_{1 t}=f(x) u_{1 x x}+u_{1} u_{1 x}-\frac{1-t}{1+t} u_{3}, \\
u_{2 t}=g(x) u_{2 x x}-u_{2} u_{2 x}+\frac{1+t}{1-t} u_{3}, \\
0=u_{1}(1+t)-u_{2}(1-t), \quad-\infty<x<\infty,-1<t<1,
\end{gathered}
$$

subject to the initial conditions

$$
u_{1}(0, x)=x, \quad u_{2}(0, x)=x, \quad u_{3}(0, x)=2 x,
$$

where $f(x)$ and $g(x)$ are analytical functions on $-\infty<x<$ $\infty$.
The integration of (55) and (56) with respect to $t$ and using the initial conditions (58) lead to

$$
\begin{aligned}
& u_{1}(t, x)=x+\int_{0}^{t}\left[f(x) u_{1 x x}+u_{1} u_{1 x}-\frac{1-t}{1+t} u_{3}\right] d t, \\
& u_{2}(t, x)=x+\int_{0}^{t}\left[g(x) u_{2 x x}-u_{2} u_{2 x}+\frac{1+t}{1-t} u_{3}\right] d t .
\end{aligned}
$$

PSM assumes once again that $u_{1}(t, x), u_{2}(t, x)$, and $u_{3}(t, x)$ can be written as

$$
\begin{aligned}
& u_{1}(t, x)=u_{10}(x)+u_{11}(x) t+u_{12}(x) t^{2}+\cdots, \\
& u_{2}(t, x)=u_{20}(x)+u_{21}(x) t+u_{22}(x) t^{2}+\cdots, \\
& u_{3}(t, x)=u_{30}(x)+u_{31}(x) t+u_{32}(x) t^{2}+\cdots,
\end{aligned}
$$

where $\quad u_{10}(x), u_{11}(x), \ldots, u_{20}(x), u_{21}(x), \ldots, u_{30}(x), u_{31}(x)$, $\ldots$ are unknown functions to be determined later on by the PSM method.

Substituting (61) and (63) into (59) and also (62) and (63) into (60), respectively, we get

$$
\begin{aligned}
\sum_{n=0}^{\infty} u_{1 n} t^{n}= & x+\int_{0}^{t} f(x) \sum_{n=0}^{\infty} u_{1 n}^{\prime \prime} t^{n} d t+\int_{0}^{t} \sum_{n=0}^{\infty} \sum_{m=0}^{\infty} u_{1 n} u_{1 m}^{\prime} t^{n+m} d t \\
& -\int_{0}^{t}(1-t) \sum_{n=0}^{\infty} \sum_{m=0}^{\infty}(-1)^{n} u_{3 m} t^{n+m} d t,
\end{aligned}
$$

$$
\begin{aligned}
\sum_{n=0}^{\infty} u_{2 n} t^{n}= & x+\int_{0}^{t} g(x) \sum_{n=0}^{\infty} u_{2 n}^{\prime \prime} t^{n} d t+\int_{0}^{t} \sum_{n=0}^{\infty} \sum_{m=0}^{\infty} u_{2 n} u_{2 m}^{\prime} t^{n+m} d t \\
& -\int_{0}^{t}(1+t) \sum_{n=0}^{\infty} \sum_{m=0}^{\infty} u_{3 m} t^{n+m} d t,
\end{aligned}
$$

where we have employed the Taylor series expansions

$$
\frac{1}{1-t}=\sum_{n=0}^{\infty} t^{n}, \quad \frac{1}{1+t}=\sum_{n=0}^{\infty}(-1)^{n} t^{n} .
$$

After integrating and standardizing the summation index, we get the following recursion formulas, from (64) and (65), respectively:

$$
\begin{aligned}
- & u_{10}+x-u_{30} t-\frac{1}{2}\left(u_{31}-2 u_{30}\right) t^{2} \\
- & \frac{1}{3}\left(u_{32}-2 u_{31}+2 u_{30}\right) t^{3} \\
- & \frac{1}{4}\left(u_{33}-2 u_{32}+2 u_{31}-2 u_{30}\right) t^{4} \\
+ & \sum_{k=1}^{\infty}\left[\frac{f(x) u_{1 k-1}^{\prime \prime}}{k}+\sum_{m=0}^{\infty} \frac{u_{1 m}^{\prime} u_{1 k-m-1}}{k}-u_{1 k}\right] t^{k}=0 \\
- & u_{20}+x+u_{30} t+\frac{1}{2}\left(u_{31}+2 u_{30}\right) t^{2} \\
+ & \frac{1}{3}\left(u_{32}+2 u_{31}+2 u_{30}\right) t^{3}
\end{aligned}
$$




$$
\begin{aligned}
& +\frac{1}{4}\left(u_{33}+2 u_{32}+2 u_{31}+2 u_{30}\right) t^{4} \\
& +\sum_{k=1}^{\infty}\left[\frac{g(x) u_{2 k-1}^{\prime \prime}}{k}-\sum_{m=0}^{\infty} \frac{u_{2 m}^{\prime} u_{2 k-m-1}}{k}-u_{2 k}\right] t^{k}=0 .
\end{aligned}
$$

From (57) we obtain

$$
\sum_{m=0}^{\infty} u_{2 m} t^{m}=(1+t) \sum_{n=0}^{\infty} \sum_{j=0}^{\infty} t^{n+j} u_{1 j}
$$

after using again the first series of (66).

After standardizing the summation index, we get a third recurrence formula from (68):

$$
u_{2 k}=\sum_{n=0}^{\infty}\left[u_{1 k-n}+u_{1 k-n-1}\right], \quad \text { where } k=0,1,2,3, \ldots
$$

From recursion formulas (67) and (69), we get the following coupled equations:

$$
\begin{aligned}
u_{10}= & u_{10}(0, x), \\
u_{11}= & f(x) u_{10}^{\prime \prime}+u_{10}^{\prime} u_{10}-u_{30}, \\
u_{12}= & f(x) \frac{u_{11}^{\prime \prime}}{2}+\frac{u_{10}^{\prime} u_{11}+u_{11}^{\prime} u_{10}}{2}+\frac{u_{31}}{2}, \\
u_{13}= & f(x) \frac{u_{12}^{\prime \prime}}{3}+\frac{u_{10}^{\prime} u_{12}+u_{11}^{\prime} u_{11}+u_{12}^{\prime} u_{10}}{3} \\
& -\frac{u_{32}+2 u_{30}-2 u_{31}}{3}, \\
u_{14}= & \frac{u_{13}^{\prime \prime}}{4}+\frac{u_{10}^{\prime} u_{13}+u_{11}^{\prime} u_{12}+u_{12}^{\prime} u_{11}+u_{13}^{\prime} u_{10}}{4} \\
& -\frac{u_{33}-2 u_{32}+2 u_{31}-2 u_{30}}{4}, \\
& +\frac{u_{32}+2 u_{30}+2 u_{31}}{3}, \\
u_{23}= & g(x) \frac{u_{22}^{\prime \prime}}{3}-\frac{u_{20}^{\prime} u_{22}+u_{21}^{\prime} u_{21}+u_{22}^{\prime} u_{20}}{3} \\
u_{22}= & g(x) \frac{u_{21}^{\prime \prime}}{2}-\frac{u_{20}^{\prime} u_{21}+u_{21}^{\prime} u_{20}}{2}+\frac{u_{31}+2 u_{30}}{2}, \\
u_{21}= & g(x) u_{20}^{\prime \prime}-u_{20}^{\prime} u_{20}+u_{30}, \\
u_{20} & u_{20},
\end{aligned}
$$

$$
\begin{aligned}
u_{24}= & g(x) \frac{u_{23}^{\prime \prime}}{4}-\frac{u_{20}^{\prime} u_{23}+u_{21}^{\prime} u_{22}+u_{22}^{\prime} u_{21}+u_{23}^{\prime} u_{20}}{4} \\
& +\frac{u_{33}+2 u_{32}+2 u_{31}+2 u_{30}}{4}
\end{aligned}
$$

$$
\begin{aligned}
& u_{20}=u_{10}, \\
& u_{21}=2 u_{10}+u_{11}, \\
& u_{22}=u_{12}+2 u_{11}+2 u_{10}, \\
& u_{23}=u_{13}+2 u_{12}+2 u_{11}+2 u_{10}, \\
& u_{24}=u_{14}+2 u_{13}+2 u_{12}+2 u_{11}+2 u_{10},
\end{aligned}
$$

From (70) through (84), we get the functions

$$
\begin{gathered}
u_{10}=x, \quad u_{11}=-x, \quad u_{12}=x, \\
u_{13}=-x, \quad u_{14}=x \cdots, \\
u_{20}=x, \quad u_{21}=x, \quad u_{22}=x, \\
u_{23}=x, \quad u_{24}=x \cdots, \\
u_{30}=2 x, \quad u_{31}=0, \quad u_{32}=2 x, \\
u_{33}=0, \quad u_{34}=2 x .
\end{gathered}
$$

Substituting (85) through (87) into series (61), (62), and (63), respectively, we get

$$
\begin{aligned}
& u_{1}(t, x)=x\left(1-t+t^{2}-t^{3}+t^{4}+\cdots\right), \\
& u_{2}(t, x)=x\left(1+t+t^{2}+t^{3}+t^{4}+\cdots\right), \\
& u_{3}(t, x)=2 x\left(1+t^{2}+t^{4}+t^{6}+\cdots\right) .
\end{aligned}
$$

After identifying the $n$th terms of the above series as $(-1)^{n} t^{n}$, $t^{n}$, and $t^{2 n}$, respectively, we conclude that series (88) through (90) admit the following closed forms:

$$
\begin{aligned}
& u_{1}(t, x)=\frac{x}{1+t}, \\
& u_{2}(t, x)=\frac{x}{1-t}, \\
& u_{3}(t, x)=\frac{2 x}{1-t^{2}},
\end{aligned}
$$

which is the exact solution of (55)-(58), where we have employed (66) and

$$
\frac{1}{1-t^{2}}=\sum_{n=0}^{\infty} t^{2 n}
$$

This case admits an alternative way to obtain the closed solution (91) by using Padé posttreatment $[58,59]$. In general 
terms, Padé technology is employed, in order to obtain solutions for differential equations, handier and computationally more efficient. Also, it is employed to improve the convergence of truncated series. As a matter of fact, the application of Padé [2/2] to series (88)-(90) leads to the exact solution (91).

\section{Discussion}

In this study we presented the power series method (PSM) as a useful tool in the search for analytical solutions for singular partial differential-algebraic equations (SPDAEs). To this end, two SPDAE problems of index-two and another of index-one were solved by this technique, leading (for these cases) to the exact solutions. For each of the cases studied, PSM essentially transformed the SPDAE into an easily solvable algebraic system for the coefficient functions of the proposed power series solution.

Since not all the SPDAEs have exact solutions, it is possible that, in some cases, the series solution obtained from PSM may have limited regions of convergence, even taking a large number of terms; our case study three suggests the use of a Padé posttreatment, as a possibility to improve the domain of convergence for the PSM's truncated series. In fact, the mentioned example showed that, sometimes, Padé approximant leads to the exact solution. It should be mentioned that Laplace-Padé resummation is another known method, employed in the literature [53] to enlarge the domain of convergence of solutions or is inclusive to find exact solutions. This technique, which combines Laplace transform and Padé posttreatment, may be used in the future research of SPDAEs.

One of the important features of our method is that the high complexity of SPDAE problems was effectively handled by this method. This is clear if one notes that our examples were chosen to include higher-order-index PDAEs (differentiation index greater than one), linear and nonlinear cases, even with variable coefficients. In addition the last example proposed the case of a system of equations containing two functions entirely arbitrary. The above makes this system completely inaccessible to numerical methods; also we add singularities, which gave rise to the name of SPDAEs.

Finally, the fact that there are not any standard analytical or numerical methods to solve higher-index SPDAEs converts the PSM method into an attractive tool to solve such problems.

\section{Conclusion}

By solving the three examples, we presented PSM as a handy and useful tool, with high potential to find analytical solutions to SPDAEs. Since, on one hand, we proposed the way to improve the solutions obtained by this method if necessary and, on the other hand, it is based on a straightforward procedure, our proposal will be useful for practical applications and suitable for engineers and scientists. Finally, further research should be conducted to solve other SPDAEs systems, above all of index greater than one, combining PSM and LaplacePadé resummation.

\section{Conflict of Interests}

The authors declare that there is no conflict of interests regarding the publication of this paper.

\section{Acknowledgment}

The authors gratefully acknowledge the financial support from the National Council for Science and Technology of Mexico (CONACyT) through Grant CB-2010-01 no. 157024.

\section{References}

[1] W. Lucht and K. Strehmel, "Discretization based indices for semilinear partial differential algebraic equations," Applied Numerical Mathematics, vol. 28, no. 2-4, pp. 371-386, 1998.

[2] W. Lucht, K. Strehmel, and C. Eichler-Liebenow, "Indexes and special discretization methods for linear partial differential algebraic equations," BIT Numerical Mathematics, vol. 39, no. 3, pp. 484-512, 1999.

[3] W. S. Martinson and P. I. Barton, "A differentiation index for partial differential-algebraic equations," SIAM Journal on Scientific Computing, vol. 21, no. 6, pp. 2295-2315, 2000.

[4] L. M. B. Assas, "Approximate solutions for the generalized KdVBurgers' equation by He's variational iteration method," Physica Scripta, vol. 76, pp. 161-164, 2007.

[5] J.-H. He, "Variational approach for nonlinear oscillators," Chaos, Solitons \& Fractals, vol. 34, no. 5, pp. 1430-1439, 2007.

[6] M. Kazemnia, S. A. Zahedi, M. Vaezi, and N. Tolou, "Assessment of modified variational iteration method in BVPs high-order differential equations," Journal of Applied Sciences, vol. 8, no. 22, pp. 4192-4197, 2008.

[7] R. Noorzad, A. T. Poor, and M. Omidvar, "Variational iteration method and homotopy-perturbation method for solving Burgers equation in fluid dynamics," Journal of Applied Sciences, vol. 8, no. 2, pp. 369-373, 2008.

[8] D. J. Evans and K. R. Raslan, "The tanh function method for solving some important non-linear partial differential equations," International Journal of Computer Mathematics, vol. 82, no. 7, pp. 897-905, 2005.

[9] F. Xu, "A generalized soliton solution of the KonopelchenkoDubrovsky equation using He's exp-function method," Zeitschrift fur Naturforschung, Section A, vol. 62, no. 12, pp. 685-688, 2007.

[10] J. Mahmoudi, N. Tolou, I. Khatami, A. Barari, and D. D. Ganji, "Explicit solution of nonlinear ZK-BBM wave equation using Exp-function method," Journal of Applied Sciences, vol. 8, no. 2, pp. 358-363, 2008.

[11] G. Adomian, "A review of the decomposition method in applied mathematics," Journal of Mathematical Analysis and Applications, vol. 135, no. 2, pp. 501-544, 1988.

[12] E. Babolian and J. Biazar, "On the order of convergence of Adomian method," Applied Mathematics and Computation, vol. 130, no. 2-3, pp. 383-387, 2002.

[13] A. Kooch and M. Abadyan, "Efficiency of modified Adomian decomposition for simulating the instability of nanoelectromechanical switches: comparison with the conventional 
decomposition method," Trends in Applied Sciences Research, vol. 7, no. 1, pp. 57-67, 2012.

[14] A. Koochi and M. Abadyan, "Evaluating the ability of modified adomian decomposition method to simulate the instability of freestanding carbon nanotube: comparison with conventional decomposition method," Journal of Applied Sciences, vol. 11, no. 19, pp. 3421-3428, 2011.

[15] S. Karimi Vanani, S. Heidari, and M. Avaji, "A low-cost numerical algorithm for the solution of nonlinear delay boundary integral equations," Journal of Applied Sciences, vol. 11, no. 20, pp. 3504-3509, 2011.

[16] S. H. Chowdhury, "A comparison between the modified homotopy perturbation method and adomian decomposition method for solving nonlinear heat transfer equations," Journal of Applied Sciences, vol. 11, no. 7, pp. 1416-1420, 2011.

[17] L.-N. Zhang and L. Xu, "Determination of the limit cycle by He's parameter-expansion for oscillators in a $u^{3} /\left(1+u^{2}\right)$ potential," Zeitschrift fur Naturforschung-Section A Journal of Physical Sciences, vol. 62, no. 7-8, pp. 396-398, 2007.

[18] J.-H. He, "A coupling method of a homotopy technique and a perturbation technique for non-linear problems," International Journal of Non-Linear Mechanics, vol. 35, no. 1, pp. 37-43, 2000.

[19] J.-H. He, "Homotopy perturbation technique," Computer Methods in Applied Mechanics and Engineering, vol. 178, no. 3-4, pp. 257-262, 1999.

[20] J.-H. He, "Homotopy perturbation method for solving boundary value problems," Physics Letters A, vol. 350, no. 1-2, pp. 8788, 2006.

[21] J.-H. He, "Recent development of the homotopy perturbation method," Topological Methods in Nonlinear Analysis, vol. 31, no. 2, pp. 205-209, 2008.

[22] A. Beléndez, C. Pascual, M. L. Álvarez, D. I. Méndez, M. S. Yebra, and A. Hernández, "Higher order analytical approximate solutions to the nonlinear pendulum by He's homotopy method," Physica Scripta, vol. 79, no. 1, Article ID 015009, 2009.

[23] J.-H. He, "A coupling method of a homotopy technique and a perturbation technique for non-linear problems," International Journal of Non-Linear Mechanics, vol. 35, no. 1, pp. 37-43, 2000.

[24] M. El-Shahed, "Application of He's homotopy perturbation method to Volterra's integro-differential equation," International Journal of Nonlinear Sciences and Numerical Simulation, vol. 6, no. 2, pp. 163-168, 2005.

[25] Y. Khan, H. Vázquez-Leal, and N. Faraz, "An efficient new iterative method for oscillator differential equation," Scientia Iranica, vol. 19, no. 6, pp. 1473-1477, 2012.

[26] D. D. Ganji, H. Babazadeh, F. Noori, M. M. Pirouz, and M. Janipour, "An application of homotopy perturbation method for non-linear Blasius equation to boundary layer flow over a flat plate," International Journal of Nonlinear Science, vol. 7, no. 4, pp. 399-404, 2009.

[27] D. D. Ganji, H. Mirgolbabaei, M. Miansari, and M. Miansari, "Application of homotopy perturbation method to solve linear and non-linear systems of ordinary differential equations and differential equation of order three," Journal of Applied Sciences, vol. 8, no. 7, pp. 1256-1261, 2008.

[28] A. Fereidoon, Y. Rostamiyan, M. Akbarzade, and D. D. Ganji, "Application of He's homotopy perturbation method to nonlinear shock damper dynamics," Archive of Applied Mechanics, vol. 80, no. 6, pp. 641-649, 2010.

[29] P. R. Sharma and G. Methi, "Applications of homotopy perturbation method to partial differential equations," Asian Journal of Mathematics \& Statistics, vol. 4, no. 3, pp. 140-150, 2011.
[30] H. Aminikhah, "Analytical approximation to the solution of nonlinear Blasius'viscous flow equation by LTNHPM," ISRN Mathematical Analysis, vol. 2012, Article ID 957473, 10 pages, 2012.

[31] H. Vázquez-Leal, U. Filobello-Niño, R. Castañeda-Sheissa, L. Hernández-Martínez, and A. Sarmiento-Reyes, "Modified HPMs inspired by homotopy continuation methods," Mathematical Problems in Engineering, vol. 2012, Article ID 309123, 19 pages, 2012.

[32] H. Vazquez-Leal, R. Castaneda-Sheissa, U. Filobello-Nino, A. Sarmiento-Reyes, and J. Sanchez Orea, "High accurate simple approximation of normal distribution integral," Mathematical Problems in Engineering, vol. 2012, Article ID 124029, 22 pages, 2012.

[33] U. Filobello-Nino, H. Vazquez-Leal, R. Castaneda-Sheissa et al., "An approximate solution of Blasius equation by using HPM method," Asian Journal of Mathematics and Statistics, vol. 5, no. 2, pp. 50-59, 2012.

[34] J. Biazar and H. Aminikhah, "Study of convergence of homotopy perturbation method for systems of partial differential equations," Computers \& Mathematics with Applications, vol. 58, no. 11-12, pp. 2221-2230, 2009.

[35] J. Biazar and H. Ghazvini, "Convergence of the homotopy perturbation method for partial differential equations," Nonlinear Analysis: Real World Applications, vol. 10, no. 5, pp. 2633-2640, 2009.

[36] U. Filobello-Nino, H. D. Vazquez-Leal, Y. Khan et al., "HPM applied to solve nonlinear circuits: a study case," Applied Mathematics Sciences, vol. 6, no. 87, pp. 4331-4344, 2012.

[37] D. D. Ganji, A. R. Sahouli, and M. Famouri, "A new modification of He's homotopy perturbation method for rapid convergence of nonlinear undamped oscillators," Journal of Applied Mathematics and Computing, vol. 30, no. 1-2, pp. 181-192, 2009.

[38] U. Filobello-Nino, H. Vazquez-Leal, Y. Khan et al., "Laplace transform-homotopy perturbation method as a powerful tool to solve nonlinear problems with boundary conditions defined on finite intervals," Computational and Applied Mathematics, 2013.

[39] M. Bayat and I. Pakar, "Nonlinear vibration of an electrostatically actuated microbeam," Latin American Journal of Solids and Structures, vol. 11, no. 3, pp. 534-544, 2014.

[40] M. M. Rashidi, S. A. M. Pour, T. Hayat, and S. Obaidat, "Analytic approximate solutions for steady flow over a rotating disk in porous medium with heat transfer by homotopy analysis method," Computers and Fluids, vol. 54, pp. 1-9, 2012.

[41] J. Biazar and B. Ghanbari, "The homotopy perturbation method for solving neutral functional-differential equations with proportional delays," Journal of King Saud University: Science, vol. 24, no. 1, pp. 33-37, 2012.

[42] M. Bayat, I. Pakar, and A. Emadi, "Vibration of electrostatically actuated microbeam by means of homotopy perturbation method," Structural Engineering and Mechanics, vol. 48, no. 6, pp. 823-831, 2013.

[43] M. F. Araghi and B. Rezapour, "Application of homotopy perturbation method to solve multidimensional schrodinger's equations," Journal of Mathematical Archive, vol. 2, no. 11, pp. $1-6,2011$.

[44] J. Biazar and M. Eslami, "A new homotopy perturbation method for solving systems of partial differential equations," Computers and Mathematics with Applications, vol. 62, no. 1, pp. 225-234, 2011.

[45] M. F. Araghi and M. Sotoodeh, "An enhanced modified homotopy perturbation method for solving nonlinear volterra 
and fredholm integro-differential equation 1," World Applied Sciences Journal, vol. 20, no. 12, pp. 1646-1655, 2012.

[46] M. M. Rashidi, M. T. Rastegari, M. Asadi, and O. A. Bég, "A study of non-Newtonian flow and heat transfer over a non-isothermal wedge using the homotopy analysis method," Chemical Engineering Communications, vol. 199, no. 2, pp. 231256, 2012.

[47] T. Patel, M. N. Mehta, and V. H. Pradhan, "The numerical solution of Burger's equation arising into the irradiation of tumour tissue in biological diffusing system by Homotopy Analysis Method," Asian Journal of Applied Sciences, vol. 5, no. 1, pp. 60-66, 2012.

[48] V. Marinca and N. Herisanu, Nonlinear Dynamical Systems in Engineering, Springer, Heidelberg, Germany, 1st edition, 2011.

[49] E. Ince, Ordinary Differential Equations, Dover, New York, NY, USA, 1956.

[50] A. Forsyth, Theory of Differential Equations, Cambridge University Press, New York, NY, USA, 1906.

[51] T. L. Chow, Classical Mechanics, John Wiley \& Sons, New York, NY, USA, 1995.

[52] M. H. Holmes, Introduction to Perturbation Methods, Springer, New York, NY, USA, 1995.

[53] U. Filobello-Nino, H. Y. Vazquez-Leal, A. Khan et al., "Perturbation method and laplace-padé approximation to solve nonlinear problems," Miskolc Mathematical Notes, vol. 14, no. 1, pp. 89-101, 2013.

[54] U. Filobello-Nino, H. Vazquez-Leal, K. Boubaker et al., "Perturbation method as a powerful tool to solve highly nonlinear problems: the case of Gelfand's equation," Asian Journal of Mathematics and Statistics, vol. 6, no. 2, pp. 76-82, 2013.

[55] U. Filobello-Nino, H. Vazquez-Leal, Y. Khan et al., "A handy exact solution for flow due to a stretching boundary with partial slip," Revista Mexicana de Física E, vol. 59, no. 1, pp. 51-55, 2013.

[56] H. Vazquez-Leal, "Exact solutions for differential-algebraic equations," Miskolc Mathematical Notes, vol. 15, no. 1, pp. 227238, 2014.

[57] B. Benhammouda and H. Vazquez-Leal, "Analytical solutions for systems of partial differential-algebraic equations," SpringerPlus, vol. 3, article 137, 2014.

[58] H. Bararnia, E. Ghasemi, S. Soleimani, A. Barari, and D. D. Ganji, "HPM-Padé method on natural convection of darcian fluid about a vertical full cone embedded in porous media," Journal of Porous Media, vol. 14, no. 6, pp. 545-553, 2011.

[59] G. A. Baker, Essentials of Padé Approximants, Academic Press, New York, NY, USA, 1975. 


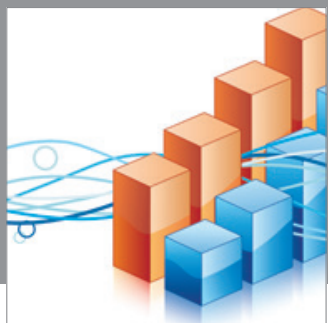

Advances in

Operations Research

mansans

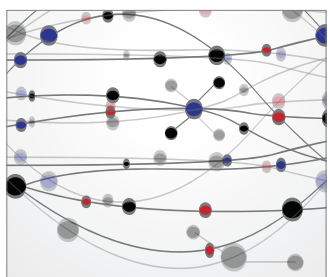

The Scientific World Journal
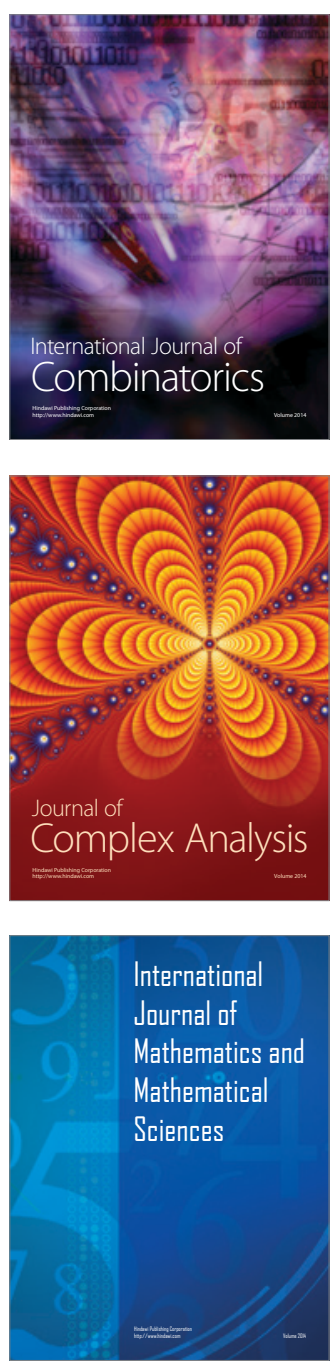
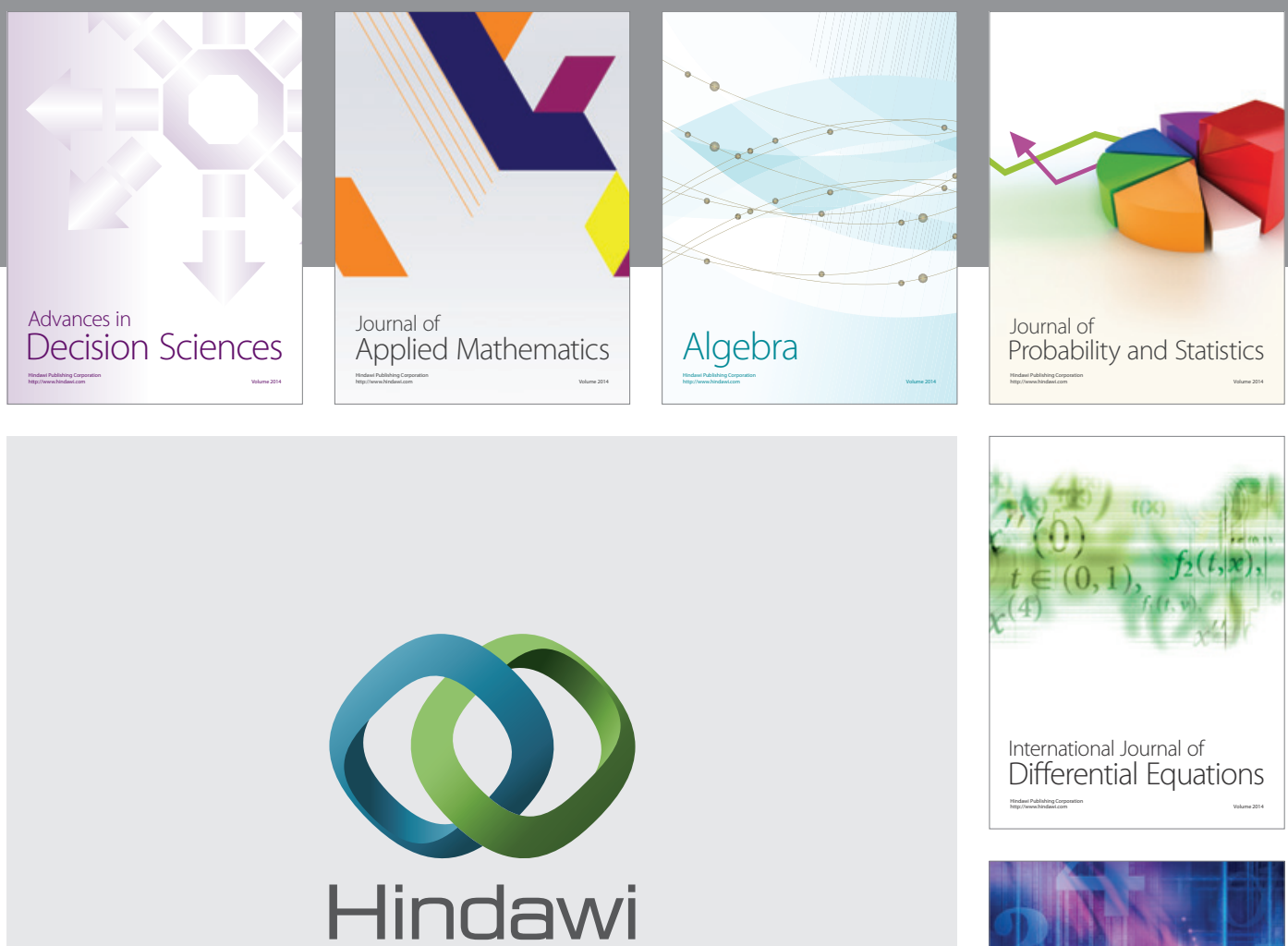

Submit your manuscripts at http://www.hindawi.com
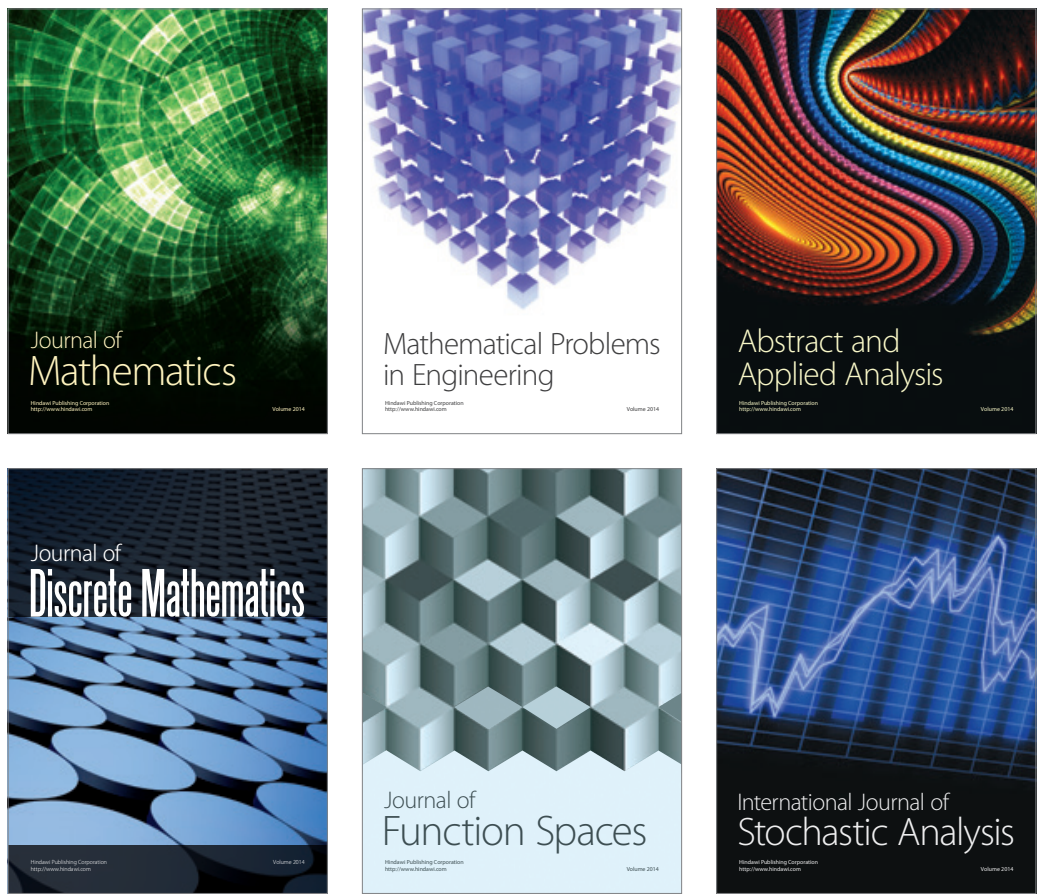

Journal of

Function Spaces

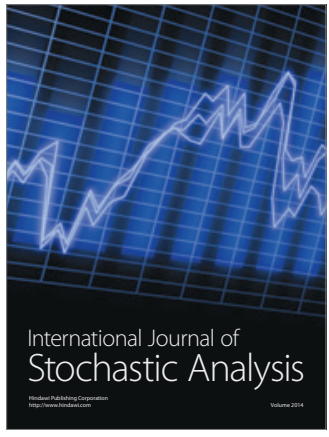

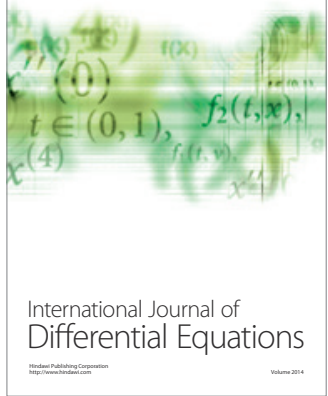
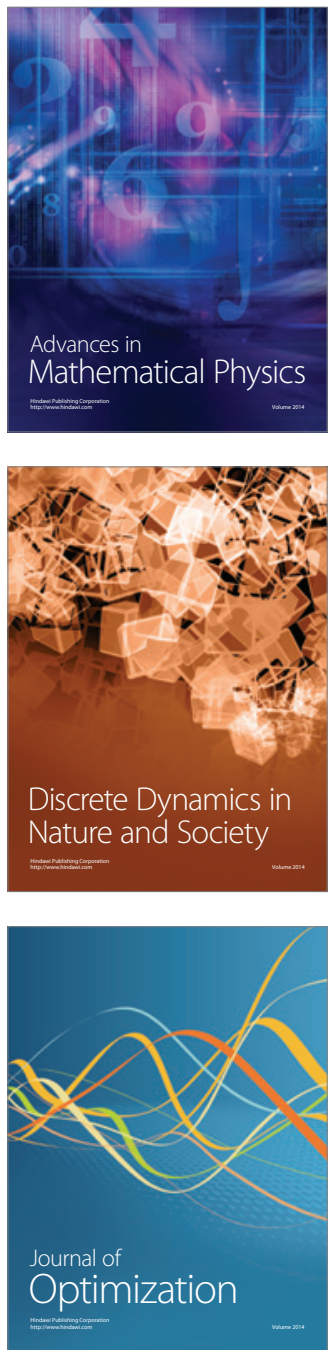